\title{
A State of Art on Energy Efficient Multipath Routing In Wireless Sensor Networks
}

\author{
Swedika Sharma \\ Department of Computer Science \& Engineering, Jaypee University of Information Technology, Waknaghat, India
}

\begin{tabular}{|c|c|}
\hline Article Info & ABSTRACT \\
\hline Article history: & Wireless sensor network is the combination of sensor nodes where sensor \\
\hline Received May 30, 2018 & $\begin{array}{l}\text { nodes are distributed all over the network. There are some challenges that } \\
\text { come into the wireless sensor network } n \text { context to energy efficiency, }\end{array}$ \\
\hline Revised Jul 25, 2018 & network lifetime, storage and battery backup. The most important feature of a \\
\hline Accepted Aug 7, 2018 & routing protocol, in order to be efficient for WSNs, is the energy \\
\hline Keywords: & $\begin{array}{l}\text { have analyzed various routing techniques for WSN that increases the } \\
\text { network lifetime and energy consumption. }\end{array}$ \\
\hline
\end{tabular}

Copyright $(2018$ Institute of Advanced Engineering and Science. All rights reserved.

\section{Corresponding Author:}

Swedika Sharma,

Department of Computer Science \& Engineering,

Jaypee University of Information Technology,

Waknaghat, India.

Email: swedika.sharma@gmail.com

\section{INTRODUCTION}

A basic understanding of computer networks is necessary in order to understand the values of network security. In this section, we will cover some of the foundations of computer networking, then move on to an overview of some popular networks. A network has been defined as any set of interlinking lines resembling a network of nodes an interconnected system, a network of alliances. This means that it suits our purpose well: a computer network is just a system of interconnected computers. Wireless sensor networks are becoming an active topic of research, where sensors are units with sensing, processing, and wireless networking capability. They can automatically collect the data and report the quantities to the sink. Recently, many wireless sensor networks have been designed and deployed for kinds of applications. An important role in many WSN operation models and applications, such as average access scheduling, information fusion, beam-forming, target tracking, etc. WSNs are used in a wide range of potential applications together with military, medical coordination and robotic exploration which explains the important attention drawn by these types of networks in research field. Since sensor nodes are usually battery powered, conserving their energy and prolonging the system life time are prime goals while designing protocols for those networks. There is very less substructure used in WSN. WSN contains large number of nodes which may vary from few thousands to obtain the information from the atmosphere. WSN is actually a type of application-oriented network, which in turn demands the single desire connected with several individual needs to be understood by simply network like it is energy saving, simplify network protocol, flexibility and autonomy. WSNs can be used for a number of applications reaching from surveillance \& atmosphere monitoring to health care and military operations. Several applications need that sensor knobs should be left unattended for a long period of time due to cost implications or difficult access to the deployment area. One of the routing technique which is quite energy efficient is PEGASIS protocol. It chooses an alternative path in WSNs. It forms a chain that 
makes the path more even-distributed and the total square of transmission distance much less. Moreover, in the constructing process, the energy factor has been taken into explanation, which carries about a balance of energy consumption between nodes. In each round of transmission, according to the current energy of each node, a leader is selected to directly communicate with the base station.

This paper is divided into three sections: Introduction in section 1, followed by related work in section 2, finally results and conclusions along with future work have been discussed in section 3 .

\section{RELATED WORK}

In this section, based on the required parameters like energy consumption, average delay, throughput, we have searched the various databases like IEEE Xplore, ACM Digital Library, Google Scholar and many more research databases. After manual review of each paper, we have considered the most relevant papers for further analysis during our related work. The considered papers are mentioned below in detail.

In [1] Barooah et al. described that, a wireless sensor network can get separated into multiple connected components due to the failure of some of its nodes, which was called a "cut". In their papers, they have considered the problem of detecting cuts by the remaining nodes of a wireless sensor network and proposed an algorithm that allows every knob to detect when the connectivity to a specially designated node has been lost, and one or more nodes (that are connected to the special node after the cut) to detect the occurrence of the cut. The algorithm was distributed and asynchronous: every node needs to communicate with only those nodes that are within its communication range. The algorithm depends on the iterative calculation of a fictitious "electrical potential" of the nodes. The convergence rate of the underlying iterative scheme is independent of the size and structure of the network.

In [2] Kadi et al. described the wireless sensor networks (WSNs) are mainly utilized in military applications due to its certainty and efficiency. Nowadays, it is considered to be the first-hand in many civilian applications such as leakage discovery. Various technical issues, like power consumption, radio propagation models and sensors are considered for different types of applications in their paper. WSN has emerged lately as an effective solution for fluid leakage. They have mentioned four different solutions for leaks in pipelines using wireless sensor networks (WSNs).

In [3] Kaur et al. described that in WSNs energy was a scarcest resource of sensor nodes and it determines the lifetime of sensor nodes. These small batteries were limited power and also may not easily rechargeable or removable. Long communication distance amid sensors and a sink can greatly drain the energy of sensors and reduce the lifetime of a network. In their work, they have reported that WSNs, energy was a big factor to be considered. Various techniques are used to optimize energy level of sensor nodes of WSN. And make a review of some of these wireless sensor network methods which are used recently and are helpful in improving energy efficiency of wireless sensor knots.

In [4] Gilbert et al. described the Wireless Sensor Networks may be used in the variety of fields which includes military, healthcare, conservational, biological, home and other profitable applications. With the huge advancement in the field of embedded computer and sensor technology, Wireless Sensor Networks (WSN), which was composed of several thousands of sensor nodes are capable of sensing, actuating, and relaying the collected information made remarkable impact everywhere. Their paper presents an overview of the various research issues in WSN based application.

In [5] Beema et al. have proposed a High Energy First (HEF) scheme in which the node that will have a high residual of energy will be chosen to be cluster heads and the remaining nodes will act as regular nodes. According to their work each and every sensor node will send the data to cluster head with which then the information will be forwarded to the base station. Following modules have been used in HEF scheme:

1. Route Discovery

2. Network Formation

3. Cluster Head Selection

4. Energy Consumption Modeling

5. Energy Efficient Routing

In [6] Masdari et al. have proposed that multipath routing may be considered as one of the solution to improve load balancing and quality of service. This is considered as one of the good method for improving the capacity of network and as well provides reliable communication. The researchers have made a comparison of the protocols and have done an analysis. Several protocols have been used to minimize the total energy consumed by the network by selecting the best path as follows:

1. Maximally Radio-Disjoint Multipath Routing (MR2)

2. Energy-Efficient Multipath Routing Protocol

3. Energy-Efficient and Collision-Aware Multipath Routing Protocol (EECA)

4. Low-Interference Energy-Efficient Multipath Routing Protocol (LIEMRO)

IJ-ICT Vol. 7, No. 3, December 2018 : 111-116 
In [7] Shwe et al. proposed an enhanced AdapCode. It was shown that this proposed scheme can guarantee the same reliability while reducing the power consumption. In wireless sensor networks there is a much need to reduce transmission of packets because the packet communication has much power or influence over consumption of power. In order to achieve it, the network coding was used as it improves network throughput. In order to find out all the neighbors, the AdapCode was enhanced by making use of the power efficient neighbor discovery protocol.

In [8] Nikolaos et al. proposed that the most important feature of a routing protocol, in order to be efficient for wireless sensor networks are: consumption of energy i.e. the energy efficiency of the sensors and the other is extension of lifetime of the network. According to their paper, the main objective of this research was to design energy-efficient nodes and protocols. Till now many energy efficient protocols have been used for wireless sensor networks and in this paper the researchers have used following routing protocols:

1. Network Structure

2. Communication Model

3. Topology Based

4. Reliable Routing

In [9] Intanagonwiwat et al. have proposed a paradigm in which the nodes are capable of wireless communication and computation. The paradigm used is directed-diffusion paradigm. Directed-diffusion saves energy by opting good paths and by processing data in networks. It can outperform in idealized traditional schemes like in omniscient multicast, each source transmits its evening along a shortest path multicast tree to all sinks. Omniscient multicast indicates the performance achievement in an IP-based sensor networks.

In [10] Bandyopadhyay et al. discussed a distributed clustering algorithm to organize sensors into clusters. With this the sensors communicate with clusterheads and then it further communicates the total information to the processing center. Then algorithm was extended into a hierarchy of clusterheads with which more of the energy was being saved as the numbers of levels were increased. The researchers have considered a future work as well in which they plan to consider a medium access protocol and carry out research how optimal probabilities will be affected of becoming a clusterhead and the run time of an algorithm as well.

In [11] Wang et al. have proposed an energy efficient and collision aware multipath routing protocol for wireless sensor networks. According to their paper, this algorithm finds two routes which are collision free using a controlled and power adjusting flooding. The data is then transmitted with minimum power. This algorithm actually resulted in good performance, transferring of data efficiently and consumption of minimum energy.

In [12] Yahya et al. described a multipath routing protocol which is robust and energy efficient. According to their work done, the traffic allocation can be examined in two ways with this protocol; in the first method to transfer the data message a single path is used among the discovered paths and then the next alternative path is opted if the cost is under a certain threshold. In second method the transmitted message is split into a number of segments which are of equal size and error correction codes based on XOR are added. To increase probability so that the packet is received at the destination without delay, the segments are transmitted to multiple paths. The researchers have compared this protocol with other routing protocols like Directed Diffusion and $\mathrm{N}$ to 1 routing protocol and have evaluated that, the REER protocol implemented by them saves more energy, has low average delay and higher packet delivery ratio.

In [13] Vidhyapriya et al. have proposed an energy efficient adaptive protocol. The scheme of multipath routing distributes traffic among multiple paths rather than routing all the traffic along a single path. This protocol uses multiple paths between source and sink. This protocol uses less energy and provides a reliable transmission. According to their results of simulation, this routing has high performance and higher node efficiency than directed diffusion. The researchers have as well have focused on future work like how the delivery of packets should be guaranteed in situations where non-uniform transmission range exists.

In [14] Sharma et al. have categorized routing protocols in three main classes as follows:

1. Path establishment

2. Network structure

3. Protocol operation

Path establishment can be further classified in proactive, reactive and hybrid. Network structure can be further classified as data centric attribute based, hierarchical and location based. Protocol operation can be further classified as query, bio-inspired, negotiation, coherent, non coherent, QOS, multipath and mobility.

In [15] Manjeshwar et al. proposed a hybrid routing protocol (APTEEN). This protocol combines the best features of proactive and reactive networks. The nodes in this network shows a picture of network at periodic intervals in a very energy efficient manner and react to time critical situations as well. The user can 
request past, present and future data from the network. The performance of this protocol have been evaluated by the researchers and observed that it gives a good performance in consumption of energy and longevity of network.

In [16] Ehsan et al. have presented a review on research challenges on energy-aware routing techniques for Wireless Multimedia Sensor networks (WMSN), and the advantages and performance issues of each routing protocol and algorithm [16]. The requirements of QOS are also listed which are as follows:

1. Latency

2. Bandwidth

3. Jitter

4. Reliability

In [17] Gandham et al. proposed an energy efficient usage of multiple, mobile base stations to increase the lifetime of wireless sensor networks. To determine the new locations for the base station, an integer line program is used. For energy efficient routing during every round, a flow based routing protocol is used. Some metrics are as well proposed which are listed below:

1. Time until the first node dies

2. Total number of messages received

3. Energy spent per round

In [18] Younis et al. have proposed a clustering approach for ad-hoc sensor networks. HEED selects cluster heads according to the hybrid of node residual energy. The nodes join clusters such that communication cost is minimized. It exploits the availability of multiple transmission power levels at sensor nodes. According to the work done by the researchers, the results of simulation shows the extension of duration of network lifetime, supports scalable data aggregation, more fault tolerant and good load balancing.

In [19] Liu et al. discussed a Security and Energy-efficient Disjoint Route (SEDR) which is secretsharing based multipath routing problem and is taken as an optimization problem. The main aim is to maximize the lifespan and network security. The SEDR scheme has been proposed by them to deliver sliced shares to the sink node with randomized disjoint multipath routes by utilizing the available energy of sensor nodes, such that the network security is maximized without decreasing the lifetime of wireless sensor networks.

In [20] Akyildiz et al. discussed the concepts of sensor networks. The flexibility, fault tolerance, high sensing fidelity, low-cost and rapid deployment are the characteristics of sensor networks that create many new application areas for remote sensing. The sensor networks needs to satisfy the constraints such as fault tolerance, scalability, cost, hardware, topology change, environment and power consumption

In [21] Ghaffari et al. discussed a new routing mechanism that combines multipath routing with network coding. According to them, it helps in decreasing the number of required paths and transmissions in wireless sensor networks. At the time of discovering routes, the control messages which are being exchanged between nodes are reduced. On the basis of their results it was observed that network coding routing is an energy efficient technique. It improves load balancing and enhances reliability

In [22] Younis et al. proposed a clustering approach for ad-hoc sensor networks. HEED selects cluster heads according to the hybrid of node residual energy. The nodes join clusters such that communication cost is minimized. It exploits the availability of multiple transmission power levels at sensor nodes. The result of simulation shows the extension of duration of network lifetime, supports scalable data aggregation, more fault tolerant and good load balancing.

In [23] Yahya et al. have proposed an energy efficient multipath routing protocol. According to them, RELAXT utilizes the relaxation effect of the battery which increases the lifetime of battery and hence increases the overall lifetime of the sensor network. During the path construction phase, to predict the best next hop, this protocol uses a link cost function that depends on current residual energy, available buffer size and link quality. To balance energy consumption, to increase the throughput as well as minimizing packet end to end delay, this protocol routes data across multiple paths. In comparison to the other protocols, RELAX achieves lower energy consumption, high throughput and long lifetime of the node.

In [24] Schurgers et al. proposed a guideline that advocates a uniform resource utilization which can be visualized by the energy histogram and develop a spectrum of new techniques to enhance the routing in sensor networks. In the first approach, packet streams are aggregated in a robust way which results in energy reduction. In second approach, it is discussed that more uniform resource utilization can be obtained by shaping the traffic flow. The network spreading techniques are as well discussed which are listed below:

1. Stochastic Scheme

2. Energy-based Scheme

3. Stream-based Scheme

In [25] Madan et al. discussed a distributed algorithm to compute an optimal routing scheme. With this, it maximizes the time at which the first node in the network drains out of energy. The problem is 
formulated as a linear programming problem and subgradient algorithms are used to solve it in a distributed manner. The resulting algorithm gives an optimal routing scheme and the lifespan of the network is increased.

In [26] Muruganathan et al. have proposed a centralized routing protocol called Base-Station Controlled Dynamic Clustering Protocol (BCDCP). This distributes the wasted energy among all the sensor nodes in order to increase the network lifetime. It utilizes the high-energy base station to perform most energy-intensive tasks. There are various other clustering based schemes as well.

1. Low-Energy Adaptive Clustering Hierarchy (LEACH)

2. LEACH centralized(LEACH-C)

3. Power-Efficient Gathering in Sensor Information Systems (PEGASIS).

Base-Station Controlled Dynamic Clustering Protocol is compared with the above schemes and on the basis of simulation results of the researchers it is observed that BCDCP reduces overall energy consumption and improves network lifetime.

In [27] Thein et al. have proposed an energy efficient cluster-head selection algorithm for adapting clusters and rotating cluster head positions so that the energy load can be distributed among all the nodes. The proposed model is an extension to the LEACH's random cluster-head selection algorithm. The results of simulation shows that this model improves load balancing and increase the lifetime of the network.

In [28] Wei et al. have proposed a distributed clustering algorithm, Energy-efficient Clustering (EC). It determines suitable cluster sizes which depend on the hop distance to the data sink. This reduces energy consumption. The authors have additionally proposed a simple energy-efficient multihop data collection protocol to evaluate the effectiveness of EC and calculate the end-to-end energy consumption. Energy efficient Clustering is suitable for any data collection protocol which focuses on energy conservation. The performance results show that in comparison to the other two protocols, HEED and UCR, EC extends the lifetime of network and achieves energy equalization.

In [29] Wei Ye et al. have proposed a new S-MAC, a medium access protocol for wireless sensor networks. To reduce energy consumption and to support self -configuration S-MAC is used. In order to reduce consumption of energy, the nodes occasionally sleep and do not work for that particular time. While the other nodes are being transmitted, it as well sets the radio to sleep. S-MAC as well applies message passing so as to reduce the time interval between the stimulation and response. This protocol helps in collision avoidance and provides good scalability as well. This protocol gives better results and there is a minimum waste of energy in comparison to IEEE 802.11. Through the experiment performed by the researchers they analyzed that IEEE 802.11 consumes $2-6$ times more energy comparatively to S-MAC. The researchers have as well tested this protocol by using it on the nodes with which the results were quite effective.

In [30] Johnson et al. have proposed a duty-cycle protocol which meets the demanding energy requirements. There are existing duty-cycle MAC protocols as well like S-MAC which are power efficient but in this paper, a new MAC protocol is introduced i.e. RMAC. RMAC exploits cross-layer routing information to allow its control frame to setup a multihop schedule for subsequent forwarding of a data frame. Each node along the forwarding path then wakes up at the correct scheduled time to allow it to receive and forward the data frame. According to their paper, the simulation results show that RMAC has improved end to end delivery latency than S-MAC. It handles traffic contention efficiently than S-MAC without sacrificing network throughput and energy efficiency.

\section{DISCUSSION}

In wireless sensor network, consumption of energy and lifetime of the nodes are the important issues. The main goal of WSN is not only to transmit the data from source to destination but also to increase the lifetime of network. WSN's are subject to node failures because of energy constraints. Multipath routing can reduce need for route updates, balance traffic load and increase data transfer rate in WSN. Multipath routing schemes is an optimal alternative to maximize lifetime of the network.

\section{FUTURE SCOPE}

There are various routing protocols in multipath routing which are energy efficient. Mainly, we concentrated on the energy efficient protocols that have been developed for WSN. There are challenges in WSN such as energy consumption, throughput and delay. Most of the current routing protocols assume that the BS and sensor nodes are immobile. New routing protocols must be dynamic and should be able to adapt to the mobile nodes network. 


\section{REFERENCES}

[1] P. Barooah, H. Chenji, R. Stoleru and T.K. Nagy, "Cut detection in wireless sensor networks," IEEE Transactions on Parallel and Distributed Systems, vol. 23, no. 3, pp. 483-490, 2012.

[2] T. Kadi, Z. Tuwaijri and A.L. Omran, "Wireless sensor networks for leakage detection in underground pipelines: a survey paper," Procedia Computer Science, vol. 21, pp. 491-498, 2013.

[3] K. Kaur and S. Waraich, "Energy Efficient Wireless Sensor Networks based on Clustering Techniques," International Journal of Computer Applications, vol. 119, no. 11, pp. 440-451, 2015.

[4] E. Gilbert, P. Kumar, B. Kaliaperumal, "Research Issues in Wireless Sensor Network Applications: A Survey," International Journal ofInformation and Electronics Engineering, vol. 2, no.5, pp. 533-542, 2012.

[5] F.M. Beema and J. Sutha, "Energy Efficient Multipath Routing in WSN," International Journal of Engineering Science and Computing, vol. 6, no.6, pp. 7913-7916, 2016.

[6] M. Masdari and M. Tanabi, "Multipath Routing protocols in Wireless Sensor Networks: A Survey and Analysis," International Journal of Future Generation Communication and Networking, vol, 6, no.6, pp. 181-192, 2013.

[7] H.Y. Shwe and F. Adachi, "Power Efficient Adaptive Network Coding in Wireless Sensor Networks," IEEE ICC, 2011.

[8] N.A. Pantazis, S.A. Nikolidakis and D.D. Vergados, "Energy-Efficient Routing Protocols in Wireless Sensor Networks: A Survey," IEEE COMMUNICATIONS SURVEYS \& TUTORIALS, vol. 15, 2013.

[9] C. Intanagonwiwat, R. Govindan, D. Estrin, J. Heideman and F. Silva,"Directed Diffusion for Wireless Sensor Networking."IEEE TRANSACTIONS ON NETWORKING, vol. 11, no. 1, 2003.

[10] S. Bandyopadhyay and E.J. Coyle, "An Energy Efficient Hierarchical Clustering Algorithm for Wireless Sensor Networks," IEEE, 2003.

[11] Z. Wang, E. Bulut and B.K. Szymanski, "Energy Efficient Collision Aware Multipath Routing for Wireless Sensor Networks." IEEE ICC, 2009.

[12] B. Yahya and J.B. Othman, "Robust and Energy Efficient Multipath Routing Protocol for Wireless Sensor Networks," IEEE GLOBECOM, 2009.

[13] R. Vidhyapriya and P.T. Vanathi,"Energy Efficient Adaptive Multipath Routing for Wireless Sensor Networks." IAENG International Journal of Computer Science, 2007.

[14] P. Sharma and I. Kaur, "A Comparative Study on Energy Efficient Routing Protocols in Wireless Sensor Networks," IJSCI International Journal of Computer Science Issues, vol. 12, no. 4, 2015.

[15] A. Manjeshwar and D.P. Agrawal, "APTEEN: A Hybrid Protocol for Efficient Routing and Comprehensive Information Retrieval in Wireless Sensor Networks," IEEE, 2002.

[16] S. Ehsan and B. Hamdaoui "A Survey on Energy-Efficient Routing Techniques with QOS Assurances for Wireless Multimedia Sensor Networks,” IEEE COMMUNICATIONS SURVEYS \& TUTORIALS, vol. 14, no. 2, pp. 480-489, 2012.

[17] S.R. Gandham, M. Dawande, R. Prakash and S. Venkatesan, "Energy Efficient Schemes for Wireless SensorNetworks with Multiple Mobile Base Stations," IEEE GLOBECOM, 2003.

[18] O. Younis, O. and S. Fahmy, "HEED: A Hybrid, Energy-Efficient, Distributed Clustering Approach for Ad Hoc Sensor Networks," IEEE TRANSACTIONS ON MOBILE COMPUTING, vol. 3, no. 4, 2004.

[19] A. Liu, C. Zhang, Z. Chen and X. Shen, "Secure and Energy-Efficient Disjoint Multipath Routing for WSN," IEEE TRANSACTIONS ONVEHICULAR TECHNOLOGY, vol. 61, no. 7, 2012.

[20] I.F. Akyildiz, W. Su, Y. Sankarasubramaniam and E. Cayirci, "Wireless sensor networks: a survey," Elseveir, pp. 393-422, 2002..

[21] A. Ghaffari and S. Babazadeh, "Multi-Path Routing Based on Network Coding in Wireless Sensor Networks," World Applied Sciences Journal, vol. 21, no. 11, pp. 1657-1663, 2013..

[22] O. Younis and S. Fahmy, "HEED: A Hybrid, Energy-Efficient, Distributed Clustering Approach for Ad Hoc Sensor Networks," IEEE TRANSACTIONS ON MOBILE COMPUTING, vol. 3, no. 4, 2004.

[23] B. Yahya and J.B. Othman, "RELAX: An Energy Efficient Multipath Routing Protocol for Wireless Sensor Networks," IEEE ICC, 2010.

[24] C. Schurgers and M.B. Srivastava,"Energy Efficient Routing in Wireless Sensor Networks," IEEE, vol.5, no.2, 2001.

[25] R. Madan and S. Lall, "Distributed Algorithms for Maximum Lifetime Routing in Wireless Sensor Networks," IEEE COMMUNICATIONS SOCIETY GLOBECOM, 2004.

[26] S.D. Muruganathan, S. Daniel, R. Bhasin and A.O. Fapojuwo, "A Centralized Energy-Efficient Routing Protocol for Wireless Sensor Networks," IEEE RADIO COMMUNICATIONS, 2005.

[27] M.C. Thein and T. Thein, "An Energy Efficient Cluster-Head Selection for Wireless Sensor Networks," IEEE International Conference on Intelligent Systems, Modelling and Simulation, 2010.

[28] D. Wei, Y. Jin, S. Vural, R. Tafazolli and K. Moessner, "An Energy-Efficient Clustering Solution for Wireless Sensor Networks," IEEE TRANSACTIONS ON WIRELESSCOMMUNICATIONS, vol. 10, no. 11, 2011.

[29] Y. Wei, J. Heidemann and D. Estrin, “An Energy-Efficient MAC Protocol for Wireless Sensor Networks,'IEEE INFOCOM, 2012.

[30] S. Du, A.K. Saha and D.B. Johnson, "RMAC: A Routing-Enhanced Duty-Cycle MAC Protocol for Wireless Sensor Networks.” IEEE INFOCOM, 2007. 